\title{
SENTENCE COMBINING EN EL AULA: UN INSTRUMENTO PARA LA ADQUISICIÓN Y DESARROLLO DE DESTREZA SINTÁCTICA DEL ESTUDIANTE DE INGLÉS
}

\author{
Ángel Sánchez Escobar
}

\section{Introducción:}

En mi artículo "La enseñanza de la composición escrita: un 'renacimiento' olvidado en el aula de inglés", publicado en 1994 en Philología Hispalensis, me refería a los diferentes modelos para la enseñanza de la composición inglesa --expresivo, epistemológico, retórico y formalista-- y me inclinaba, por las peculiares características de nuestros alumnos de segunda lengua, por el último, por el modelo formalista, cuyo énfasis radicaba en el texto y en la enseñanza de la oración como base para la adquisición de pericia en la composición del párrafo y del ensayo.

Con respecto al método formalista ofrecía unos breves apuntes referentes a prácticas de clase como las llamadas "generative rhetoric" (retórica generativa) y "sentence combining" (combinación de oraciones). Aunque ambas opciones resultan de gran utilidad en el aula de inglés, en este artículo me centraré en la práctica de combinación de oraciones, que representa, como lo avala una abundante investigación, un medio muy eficaz para la adquisición y desarrollo de destreza sintáctica.

$\mathrm{Y}$ aunque esta investigación se ha hecho principalmente en la adquisición del inglés como lengua nativa, hay muchas razones --incluyendo mi experiencia personal en el aula-que indican que dicha práctica podría tener relevancia en la adquisición del inglés como segunda lengua --y, sin lugar a dudas, la del español como lengua nativa--.

Como dijo Strong (1976), uno de los principales teóricos en este campo, la combinación de oraciones es una vuelta a lo básico para ir más allá. Es decir, por un lado se vuelve a la oración "kernel" y a la idea de transformación, se acude al potencial lingüístico almacenado en cada cual, sin añadir más datos al cerebro, sin análisis estructurales, sin diagramas, sino con el fin de explorar y activar esta capacidad lingüística para generar nuevas secuencias oracionales. Es decir, de dos oraciones tan básicas como:

A. The man made the people scream

The man ate fire. ( + ing)

el estudiante va ser capaz de construir oraciones tan complejas como:

B. The fire-eating man made the people scream. 
Por otro lado, puesto que esta práctica entrena al alumno a retener secuencias cada vez más largas de discurso, significa más que una vuelta a lo básico, una manera de intervenir en el desarrollo cognoscitivo, y quizás de fomentarlo. Es por ello por lo que la combinación de oraciones cobra sentido en el desarrollo sintáctico del alumno como medio para escribir, hablar y razonar en la lengua con creciente complejidad y competencia, sin necesidad de instrucción gramatical formal. Aquí la gramática no se ve como producto sino como proceso; es decir, a través de los ejercicios en la combinación de oraciones, el alumno va a ser capaz de construir la gramática desde su propio repertorio lingüístico.

\section{Historia, teoría y etapas de la combinación de oraciones:}

"Sentence Combining" nació como la aplicación de la teoría generativa y tranformacional a la enseñanza de la escritura en un momento en el que, como algunos estudios habían confirmado (Braddock, 1963), se dudaba de la eficacia de la instrucción formal gramatical en la escritura. La práctica de combinación de oraciones tiene, pues, una deuda clara con Syntactic Structures de Noam Chomsky en 1957 y con el desarrollo posterior de esta teoría por Lees en 1960 y 1961, y por el mismo Chomsky en 1965. (O’Hare 1973: 5-6).

Como es de conocimiento general, la lingüística generativa y transformacional revolucionó la teoría gramatical al formular, a través de los hechos de la actuación, el sistema lingüístistico que subyace en el hablante, es decir su competencia, e identificarla con un sistema de reglas gramaticales, coherentes y sucintas, cuyo cumplimiento permitía al hablante actuar lingüísticamente. Según esta premisa, la gramática se concebía como un mecanismo capaz de generar oraciones a través de distintas transformaciones. El generativismo demostraba que el hablante de una lengua domina inconscientemente el sistema de reglas gramaticales que hace posible su actuación lingüistica (López Morales, 1974: 28-31)

Por otro lado, Chomsky y sus seguidores demostraron la superioridad de ciertos aspectos de la gramática transformacional sobre los de la gramática tradicional, y obligaba a replantear trescientos años de instrucción gramatical. En la gramática tradicional, la principal preocupación había sido la descripción de los niveles profundos y superficiales de la estructura de la lengua y la relación entre éstos. En la gramática estructuralista, el interés había sido la descripción del nivel superficial. En la gramática transformacional se volvía en cierta manera a la gramática tradicional pero con un cambio importante: el objetivo era la descripción del proceso mediante el que la estructura profunda se transformaba en la estructura superficial (Weaver, 1979: 104).

La teoría de la combinación comienza a desarrollarse en distintas etapas en este contexto de la linguística generativa y transformacional, tomando como base los conceptos de competencia/actuación, tranformación y de una nueva teoría gramatical. La primera etapa corresponde a la aportación de John C. Mellon (1967). Él mismo cuenta que después de leer English Sentences de Paul Roberts, el primer libro de gramática que incorporaba 
transformaciones, tuvo la idea de invertir el orden de estas transformaciones dividiéndolas en cinco, seis, siete o incluso más oraciones cortas, cada una individualmente acompañadas con indicadores que indicaran reglas de transformación, para guiar al estudiante a reconstruir la oración compleja de la que estas procedían (Mellon, 1979: 2-3). Durante ocho meses, Mellon investigó con un grupo experimental al que expuso a la práctica de la combinación de oraciones junto con instrucción en gramática transformacional, y descubrió que el grupo experimental conseguía un desarrollo sintáctico en sus escritos, medido en el crecimiento de "T-units"1, veinte veces mayor que el grupo de control y que además este grupo disfrutaba con los problemas de combinación de oraciones por el reto intelectual que representaban.

La segunda etapa corresponde a la aportación de Frank O’Hare, en 1971, que desarrolla la teoría de Mellon. Lo que hace O`Hare es sustituir los indicadores que representaban las reglas de transformación, y que obligaban al estudiante a memorizar muchas instrucciones gramaticales, por la palabra misma o por indicadores que no necesitaban ninguna instrucción en gramática tradicional o transformacional. Veamos un ejemplo del mismo ejercicio en ambos autores:

[Mellon]

A1. SOMETHING took real courage.

Senator Skinner asserted SOMETHING. (T: infin--T: exp)

He didn't care (about) SOMETHING. (T: fact)

The voters thought something of him. (T: wh)

[O'Hare]

A2. SOMETHING took real courage.

Senator Skinner asserted SOMETHING. (IT-FOR-TO)

He didn't care (about) SOMETHING. (THAT)

The voters through something of him. (WHAT)

B. It took real courage for senator Skinner to assert that he didn't care what the voters thought of him.

En A2, O`Hare sustituye (T: infin), por (FOR-TO); (T: expletive) por (IT), (T: fact) por (THAT), y (T: wh), que podía ser "who", "what", "when", "where", o "why", por la palabra a usar (WHAT). O’Hare dejó SOMETHING que era un indicador de posición nominal.

En sus investigaciones O’Hare llega a conclusiones similares, es decir, a la relación directa entre la práctica en combinación de oraciones y el desarrollo sintáctico en la escritura de los estudiantes, pero sin necesidad de instrucción formal en gramática. La única instrucción que sus estudiantes recibían era la referente a cómo se debían combinar las

\footnotetext{
${ }^{1}$ Una "T-unit" consiste en una oración principal más una subordinada o forma no finita que a menudo la sustituye.
} 
oraciones. Con esta base teórica, O`Hare realizó su propio libro de ejercicios llamado Sentencecraft, publicado en 1974 . Veamos algunos ejercicios:

\section{LESSON SEVEN: THAT and THE FACT THAT}
A. Peter noticed SOMETHING.
There were nine golf balls in the river. (THAT)
B. Peter noticed that there were nine golf balls in the river.
A. SOMETHING should make you avoid him.
$\mathrm{He}$ is an absolute nut. (THE FACT THAT)
B. The fact that he is an absolute nut should make you avoid him.

\section{LESSON EIGHT: IT- THAT}
A. And SOMETHING came to pass.
Cain brought an offering unto the Lord (IT-THAT)
B. And it came to pass that Cain brought an offering unto the Lord.
A. Something occurred to Captain Sharp.
His men did not know SOMETHING. ( IT- THAT)
They were sailing through a mined area. (THAT)
B. It occurred to Captain Sharp that his men did not know that they were sailing through a mined area.

En este libro el profesor, al principio, era el centro de la actividad ya que tenía que explicar los ejercicios hasta que el alumno comprendiera la dinámica. Poco a poco, los ejercicios se hacían cada vez más complejos. En la siguiente lección, el alumno no sólo tenía que expandir sino suprimir ciertos fragmentos. Veamos la lección veintidós:

LESSON TWENTY-TWO: Underlining as a Combining Signal.
A. Miss Jones easily smeared her attacker.
Miss Jones was a former wrestler.
B. Miss Jones, a former wrestler, easily smeared he attacker. 
A. SOMETHING is impossible.

A chef cooks meals. (IT-FOR-TO)

The chef is working in this small kitchen.

The meals will satisfy all customers. (WHICH/THAT)

B. It is impossible for a chef working in this small kitchen to cook meals that (which) will satisfy all customers.

O`Hare observó que para los alumnos era un reto enfrentarse a los ejercicios de combinación de oraciones, y que hacía que entre ellos surgiesen diversas opiniones de cual era la mejor solución.

Una tercera etapa corresponde a las investigaciones de William Strong que culminó con la publicación en 1973 de Sentence Combining: A Composing Book. Strong eliminó todos los indicadores de transformación, y creó lo que se llamó el "formato abierto" (open format). El tipo de ejercicios que Strong proponía se adaptaba más a la composición de discursos completos, y se requería un mayor desarrollo sintáctico del inglés; el resultado de este ejercicio, al eliminarse todo indicativo de transformación, era distinto para cada alumno. Veamos un ejemplo:

\section{ROCK CONCERT}

1. The singer was young.

2. The singer was swarthy.

3. He stepped into the spotlight.

3. The spotlight was red.

5. His shirt was unbuttoned.

6. The unbottoned shirt bared his chest.

7. Sounds ballooned around him.

8. The sounds were of guitars.

9. The sounds were of drums.

10. The sounds were of girls.

11. The girls were screaming. 
Como observamos en este ejercicio no hay ningún indicador que señale al estudiante cómo debe unir las oraciones, ni qué oración debe servir como base para la incrustración o expansión de las oraciones. Si combinamos los tres grupos iniciales de oraciones, podríamos tener algo así:

The singer, who was young and swarthy, stepped into the red spotlight. His unbuttoned shirt bared his chest. Sounds of guitar, drums, and screaming girls ballooned around him.

Pero también podríamos tener otras versiones. En ésta se usan diferentes transformaciones para dar una oración acumulativa:

As the sounds of guitars, drums, and screaming girls ballooned around him, the young, swarthy singer stepped into the red spotlight, his shirt unbuttoned, baring his chest.

Aquí no hay soluciones predeterminadas. Con este tipo de ejercicios se obligaba al estudiante a buscar diferentes posibilidades de combinaciones y transformaciones.

La cuarta etapa en el desarrollo de la práctica de la combinación de oraciones consistió en su aplicación con el mismo "formato abierto" de Strong a un curso completo de composición. Este fue el caso de The Writer's Options: College Sentence Combining (1979), de Daiker, Kerek y Morember, que incluía completas explicaciones gramaticales:

\section{Basic Pattern Sentence}

Combine each of the groups of sentences below into a single sentence containing at least one absolute.

EXAMPLE:

1. When I walked in, Grandpa was sitting at the kitchen table.

2. The newspaper was spread before him.

When I walked in, Grandpa was sitting at the kitchen table, the newspaper spread before him.

Observemos ahora ejemplos de la última edición de este libro (1994). Los autores han preparado ejercicios de relativo, pero antes de pedir al estudiante que los realice, emplean diez páginas en explicar, siempre mediante el uso de la combinación de oraciones, cómo se forma una oración de relativo, en qué ocasiones hay que usarlas y qué normas de puntuación hay que seguir:

\section{$\underline{\text { Relative Clauses }}$}

I. Make each sequence below into a single sentence by converting the marked sentence into a relative clause. Decide whether the clause needs to be separated from the sentence by a comma.

EXAMPLES

1. A weak economy can cloud the job prospects of college graduates. 
A weak economy discourages older workers from retiring.

A weak economy that discourages older workers from retiring can cloud the job prospects of colleges graduates.

2. The students had received straight A's for the semester.

The college honored the students.

The students whom the college honored had received straight A's for the semester.

The students that the college honored had received straight A's for the semester.

The students the college honored had received straight A's for the semester.

Otra etapa en este desarrollo de la teoría y práctica de la combinación de oraciones se da en 1979 con Cooper. En su "An Outline for Writing Sentence-Combining Problems", Cooper no acude a explicaciones gramaticales a la manera tradicional sino, por su deuda con la teoría generativa, a la gramática concebida como proceso (Weaver, 122-124). Así pues, nos muestra técnicas para la preparación de ejercicios específicamente en la adjetivación -concepto fundamental para la gramática transformacional-- y en la nominalización. Veamos algunos casos:

I. Noun Modifiers

A. Adjetive words embeddings.

1. Simple

a. before subject.

The canary flew out the window.

The canary is yellow.

The yellow canary flew out the window.

2. Participle

a. ing

He saw the dog.

The dog sleeps.

He saw the sleeping dog.

II. Noun Substitutes

1. Fact clauses

SOMETHING pleased him.

It snowed. (the fact that)

The fact that it snowed pleased him. 
2. Question clauses

SOMETHING made her angry.

She read something in the note. (what)

What she read in the note made her angry.

Cooper ofrecía gran número de claves para la preparación de ejercicios en la combinación de oraciones, y tendría mucha influencia en muchos textos posteriores; sin embargo, no mencionaba casos de adverbialización. Otros teóricos como Temple y Gillet (1989) lo harán ${ }^{2}$ :

\section{A. Subordinate Clauses}

The children went to the ball game.

They washed the dishes. (after)

After they washed the dishes the children went to the ball game.

En textos como Sentence Combining. Practice Plus (Book 1: 1984 y Book 2: 1985) Donald, Orsini y Wagner, además de estructurar toda la práctica de la combinación de una manera muy clara, y con abundante práctica en oraciones subordinadas, ofrece transformaciones para combinar verbos, objetos, sujetos y predicados. Veamos algunos ejemplos:

1. The dog chased the squirrel.

The cat chased the squirrel

The dog and the cat chased the squirrel.

2. The firm built the hospital.

The office building was erected by the firm.

The firm built the hospital and the office building.

Otra novedad de Sentence Combining. Practice Plus es la aplicación de la combinación de oraciones para la enseñanza del párrafo, aunque no lo hace más que a nivel de la llamada "topic sentence", u oración temática, generalmente la primera del párrafo. Su propuesta es "combinar" información en esta oración inicial, lo que influirá en el planteamiento y desarrollo del párrafo. Es decir, cada una de estas oraciones temáticas resultará en un párrafo diferente:

1. The guitar player uses this instrument. (Noun-Verb-Noun)

2. The guitar player and the drummer use these instruments. (Compound Subject)

\footnotetext{
${ }^{2}$ Temple y Gillet aconsejan la práctica de la combinación de oraciones para enseñar a niños a escribir composición.
} 
3. The guitar player and the drummer use these American-made instruments. (Adjective)

4. In private, the guitar player and the drummer, both from England, use these American- made instruments. (Prepositional Phrase)

En definitiva, todos los proponentes de la combinación de oraciones tales como Mellon, O’Hare, Strong, Daiker, Kerek, Moremberg y Cooper, basaron sus ejercicios en el paradigma de la gramática generativa de dos formas importantes: 1) la relación entre competencia y actuación para la adquisición y empleo de su capacidad lingüística, y 2) el concepto de transformaciones, que permite insertar o incrustrar una oración en otra.

La primera distinción entre competencia y actuación puede ya de por sí justificar el uso de este tipo de ejercicios para desarrollar la capacidad sintáctica de los estudiantes, puesto que se les da la oportunidad de producir mediante la combinación de oraciones básicas ("kernels") oraciones más complejas y variadas. Es decir, con la práctica de combinación de oraciones, los estudiantes van a ser capaces de transformar en actuación real su competencia linguïstica.

El segundo concepto chomskiano, la transformación, explicaba por qué una oración se podía combinar con otra. La idea de la transformación daba cuenta de la desaparición de partes de las originales oraciones "kernel" al combinarse entre sí o incrustarse unas en otras. La teoría de que una oración pasa por algunos cambios estructurales o se transforma al pasar de su forma original (estructura profunda) a su forma final (estructura superficial) explica por qué todas las partes de dos o más oraciones dadas existen mientras que otras partes no aparecen en la estructura superficial. Realmente la gramatica transformacional daba razones coherentes de cómo funciona la combinación de oraciones y la razón de su utilidad para el desarrollo de la fluidez sintáctica sin instrucción gramatical.

\section{Aplicación a la adquisición del inglés como segunda lengua}

La primera cuestión a plantearse debe estar relacionada con la aplicación de la práctica de combinación de oraciones en nuestras aulas de inglés para impulsar el desarrollo sintáctico de los estudiantes. Una primera respuesta, partiendo de los conceptos y ejemplos expuestos hasta ahora y de mi propia experiencia, sería afirmativa. Hay que tener en cuenta, por un lado, que nuestros alumnos, en distinto grado, sí van a tener suficiente conocimiento del inglés como para comprender las oraciones básicas que sirven para las transformaciones; y que, por otro lado, por las características de su primera lengua, el español, también van a ser capaces de re-conocer intuitivamente e incorporar a su complejo mecanismo cognoscitivo el proceso de adjetivación, nominalización y adverbialización propio de estas transformaciones.

Es indudable también que el conocimiento intuitivo que tienen como hablantes de una primera lengua, aunque en un primer momento ocasione un inevitable número de errores, va a resultarle crucial para comprender los problemas sintácticos con los que se van a encontrar. Además, al igual que en las otras lenguas romances, en español existen conceptos 
gramaticales, equiparables al inglés, relativos a las partes del discurso y de la oración; es decir, con poca instrucción, van a poder identificar sustantivos, verbos, adjetivos, adverbios, preposiciones, pronombres, sujetos, predicados, objetos, etc, cuyo reconocimiento va a ser importante al construir las transformaciones.

Una segunda cuestión a plantearnos debe estar relacionada con las características del estudiante receptor de esta práctica. Aquí tendríamos que considerar que por su carácter de instrucción en el aula, la combinación de oraciones hace que los estudiantes tengan que participar en la resolución de problemas de origen sintáctico, en complejas tareas de deducción y de memorización, por lo que va a exigir de ellos cierta madurez cognoscitiva y algún conocimiento metalingüístico --saber lo que es un sustantivo, un verbo, un sujeto, etc (Lightbown y Spada, 1993: 19). Nuestros estudiantes de inglés como segunda lengua, de acuerdo con los niveles en los que nos movamos, sí poseen esta madurez cognoscitiva, y, con alguna instrucción gramatical según los casos, podrán adquirir sin ninguna dificultad ese conocimiento metalingüístico.

En relación a otra cuestión tan importante como el factor afectivo del estudiante de segunda lengua, se debe puntualizar que en la combinación de oraciones no se le exige que suministren vocabulario o contenido, lo que hará no se sienta bajo presión. Así se encontrará motivado y confiado para la interacción con otros estudiantes por la resolución de los problemas sintácticos que se le vaya presentando. En cierta manera, se van a dar cuenta de que son, llamésmole así, una especie de genios lingüísticos, en esa segunda lengua, lo que favorecerá su actitud hacia el inglés.

De igual manera, la práctica en la combinación de oraciones ayuda al desarrollo cognoscitivo al hacer que realicen, tanto escrito como oral, ejercicios cada vez más complejos y que tengan que retener en la memoria secuencias lingüísticas cada vez más largas. Otro factor importante en este sentido es que las oraciones transformadas se pueden visualizar al escribirlas en la pizarra o a través del proyector de transparencias, y en muchos casos hacer que opinen respecto a otras soluciones.

En mi opinión, la práctica de combinación de oraciones crea un ambiente en la clase relativamente cercano a la adquisición natural de la sintaxis, sin prácticamente instrucción formal $^{3}$, ya que aunque programadas las actividades resultan fáciles de realizar al trabajarse con unidades oracionales básicas que el alumno no tiene que componer. Por esta razón, el alumno, en un principio guiado por el profesor y con una mínima corrección, va a poder concentrarse en la interiorización de las reglas sintácticas. De igual manera, al ser sintáctico el foco exclusivo de los ejercicios, el alumno no va a tener dificultad en asimilar reglas de puntuación, ortografía, concordancia, paralelismo, que va a comprender se necesitan para darle una mejor "apariencia" al texto.

\footnotetext{
${ }^{3}$ Según Ellis (1985: 246) está aún por demostrar el efecto de la instrucción formal en la adquisición de una segunda lengua.
} 
Como la combinación de oraciones procede de la lingüística generativa y transformacional, podíamos plantearnos con Krashen --proponente de las teorías de Chomsky a la adquisición de una segunda lengua-- algunas cuestiones algo más abstractas. Desde el punto de vista de la "creative construction theory", el alumno adquiere una segunda lengua al leer y oír, y comprender, secuencias oracionales. Así pues, y de acuerdo con Kreshen (1982), la adquisición de la fluidez sintáctica y su repercusión en la escritura y en la comunicación oral, no resultaría de la práctica en sí sino de la comprensión de la misma mecánica de combinar oraciones. Es decir la práctica sería una consecuencia de la incorporación de los nuevos conocimientos a su capacidad cognoscitiva. Aunque, como es de suponer, nos movemos en un terreno que está por investigar y demostrar.

He dejado para el final la elección del formato, con indicador o sin indicador. Esta decisión dependerá de qué concepto sintáctico se esté practicando. Como regla general, a medida que el alumno adquiera ese desarrollo sintáctico, menos falta harán estos indicadores porque nos estaremos acercando a la composición libre de párrafo y de ensayos completos. Por supuesto, aunque la aportación de Mellon es indudable, no creo que deban llevar indicadores con reglas de transformación. En este sentido, lo que realmente ayuda a nuestros alumnos es la división clara de los ejercicios en parcelas reducidas y definidas sintácticamente.

\section{Para profesores de lengua: ventajas y resultados}

Veamos algunas ventajas y resultados de la aplicación de la combinación de oraciones en nuestras aulas de inglés, especialmente para la composición escrita. Entre las ventajas podríamos destacar que los alumnos:

1. pueden realizar ejercicios previamente programados y preparados por el profesor de acuerdo con sus necesidades, como complemento del libro de texto o en sesiones especiales;

2. pueden practicar la escritura sin que el profesor tenga que dedicar mucho tiempo para la corrección o evaluación de lo que producen;

3. puesto que pueden realizar los ejercicios con relativa facilidad, favorece su interacción con los demás en el aula;

4. descubren maneras alternativas de decir cosas y los pros y contras de un determinado modelo de oración;

5. encuentran ya un contenido y no tienen que "extraerlo" de su reducido vocabulario;

6. pueden concentrarse más en reglas ortográficas al no tener que componer las oraciones.

Entre los resultados podríamos hacer resaltar la:

1. la fluidez sintáctica: escribir más y más fácilmente; 
2. el desarrollo sintáctico: escribir oraciones de mayor complejidad;

3. la diversidad sintáctica: escribir usando muchos modelos;

4. el reconocimiento sintáctico: conciencia de la naturaleza de las oraciones;

5. la revisión de su actuación linguística: poder reordenar oraciones elementales en formas más efectivas.

6. conciencia de la relación entre sintaxis y el significado. 


\section{BIBLIOGRAFÍA}

Braddock, R., Lloyd-Jones, R. y Schoer, L. 1963. Research in Written Composition.

Urbana, Ill.: National Council of Teachers of English.

Cooper, Charles. 1984. "An Outline for Writing Sentence-Combining Problems".

Rhetoric and Composition: A Sourcebook for Teachers, pp. 122-128. Rochelle Park, N.J.: Hayden Book Company, Inc.

Daiker, Kerek y Moremberg. 1979. The Writer's Options: College Sentence Combining. New York: Harper and Row.

Daiker, Kerek, Moremberg y Sommers. 1994. The Writer's Options: Combining to Composing. New York: Harper Collins College Publishers.

Ellis,Rod. 1985. Understanding Second Language Acquisition. Oxford University Press.

Krashen, S. 1982. Principles and Practice in Second Language Acquisition. Oxford: Pergamon.

Lightbown, Patsy M. y Spada, Nina. 1993. How Languages are Learned. Oxford University Press.

López Morales, Humberto. 1974. Introducción a la Lingüística Generativa. Madrid: Romania.

McDonald, Orsini y Wagner. 1985. Sentence Combining: Practice Plus. Newton, Mss.:

Allyn and Bacon, Inc.

Mellon, J.C. 1967. Transformational Sentence Combining: a Method for Enhancing the development of syntactic fluency in English Composition. Cambridge: Harvard University. US Office of Education Cooperative Research Project 5-8418.

Mellon, J.C. 1979. "Issues in the Theory and Practice of Sentence Combining". SentenceCombining and the Teaching of Writing,Daiker, Kerek y Morember, eds. Conway, Ark.: University of Central Arkansas.

O’Hare, Frank. 1973. Sentence Combining: Improving Student Writing without Formal Grammar Instruction. Urbana, Ill.: National Council of Teachers of English.

O`Hare, Frank. 1974. Sentencecraft. Ginn.

Strong, William. 1973. Sentence Combining: A Composing Book. Urbana, Ill.: National Council of Teachers of English.

Temple, Charles y Gillet, Jean Wallace. 1989. Language Arts. Learning Processes and Teaching Practices. Harper Collins Publishers

Weaver, Constance. 1979. Grammar for Teachers: Perspectives and Definitions.Urbana, Ill.: National Council of Teachers of English. 


\section{APÉNDICE: NOTAS PARA LA PREPARACIÓN DE EJERCICIOS DE COMBINACIÓN DE ORACIONES}

A continuación voy a proponer una serie de ejercicios que se pueden ajustar a diferentes niveles. Pero antes de comenzar con práctica en combinación de oraciones --y lo que hemos denominado gramática como proceso--, creo que sería necesario que el alumno re-conociera las partes del discurso primero, es decir, sustantivos, verbos, adjectivos, preposiciones y pronombres, para después, también como paso previo, continuar con el re-conocimiento de las partes de la oración. (Muchos ejercicios vienen sin realizar para la propia práctica del profesor).

\section{PARTS OF SPEECH}

Directions: In each of the following sentences two words are italicized. Below each italicized word write one of the following numbers and then copy the numbers in the right order in the spaces at the left:

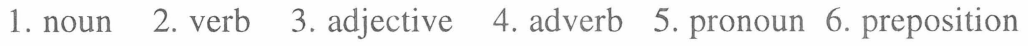

Example:

214 I want to talk to your brother now.

1. The students are kind with the new English teacher.

2. My family lived in New York for five years.

\section{SIMPLE SENTENCE PATTERNS}

Previamente también se les debería instruir y hacer práctica en la oración simple y su funcionamiento, incluidos los diferentes modelos de oraciones. Las explicaciones que demos en este respecto deben ser sucintas y claras, por ejemplo (Orsini y Wagner): 
Grammar defines a simple sentence as a group of words that contains a subject and a predicate. The subject is a word or a group of words (often a noun or a pronoun). The predicate is the verb and all its modifiers and objects. Example: John plays soccer

Types of sentences: 1 . declarative 2 . interrogative 3 . imperative

Types of verbs: action, linking, and auxiliary

Action verbs: 1. transitive (The film bored us) 2. intransitive (Intermission came) Linking verbs: be (is expensive), seem, grow, look, prove, remain, appear

Auxiliary verbs: be (is coming), do, does, did, can, could, would, should, shall, will, etc Basic Sentence Patterns:

1. N--V The boys laughed NS IntV

2. N--V--N Jim sang the blues

NS TrV NDO

3. N--V--N--N Tom gave Mary a present

NS TraV NIO NDO

Everybody thinks the man a genius NS TraV NDO NOC

4. N--A--V--[N] The man was killed by the tiger NS A $\mathrm{V}$ by

5. N--LV--N She was my assistant NS LV NSC

6. N--LV--Adj The boy seems unhappy NS LV AdjSC

\section{Activity 1: Sentence Patterns}

Directions: On a separate sheet of paper, copy the sentences below. Then label the different parts of the sentences and give the pattern number:

Example: I will pay the check

NS

A

$\operatorname{Tr} \mathrm{V}$

NDO

Pattern 2

1. I am not a polluter.

Pattern -

\section{Activity 2: Subject and Predicate}

Directions: On a separate sheet of paper, copy the following sentences. For each sentence underline the subject with a single line and the predicate with a double line. Then indicate whether the sentence is declarative, interrogative, or imperative. With an imperative sentence you will have to insert the understood "you" in parentheses.

Examples: The film was interesting. declarative

1. Give her the chocolate bar.

2. Everyone will fail English.

Una vez que han aprendido a identificar las partes de la oración, pueden comenzar con la práctica en combinación de oraciones: 
Activity 3: Combining Subjects, Objects, and Complements

Directions: On a separate sheet of paper, make a sentence with a compound subject, a compound object or a compound complement out of each group below.

1. Peter joined the basketball team.

Steve joined the basketball team.

Peter and Steve joined the basketball team.

2. Mr. Baker gave his daughter money.

Mr. Baker gave his son money.

3. My best friend seemed a teacher

He appeared a scholar too.

My best friend seemed a teacher and a scholar.

\section{Activity 4: Combining Predicates}

1. They did go to the party.

They had a good time.

They returned home very late.

They did go to the party, had a good time, and returned home very late.

Las actividades de combinación de oraciones que siguen necesitarían de igual manera una explicación de su uso, forma y posición en la oración.

\section{Activity 5: Combining Adjectives}

1. My sister spent two hours in the store.

The store had only secondhand books.

The store was big.

My sister spent two hours in the secondhand book store

Activity 6: Combining Adverbs:

1. The dog is drinking the water in the bowl. 
$\mathrm{He}$ is drinking noisily.

He is drinking quickly.

The dog is noisily and quickly drinking the water in the bowl.

2. Joanna played in a jazz band.

She did it frequently.

She played late at night.

Joanna frequently played late at night in a jazz band.

\section{Activity 7: Combining Prepositional Phrases}

1. Jim had an accident.

The accident happened at an intersection

Jim had an accident at an intersection.

2. Follow the guide.

$\mathrm{He}$ is going to the Science Museum.

Follow the guide to the Science Museum.

3. Gary received a ticket.

The ticket was for parking.

He parked in front of a hospital.

The meter maid gave him the ticket.

Una vez que el alumno haya practicado con los elementos básicos de la oración simple, puede pasar a la oración compuesta. Aquí se pueden realizar innumerables operaciones con combinación de oraciones ya sean con indicador o sin indicador. Un completo cuadro de las conectivas junto con sus reglas de puntuación puede servir para hacer tipos variados de actividades. 
CONNECTIVES USED TO EXPRESS DIFFERENT RELATIONSHIPS BETWEEN IDEAS

Types of relationships
Compound

Sentences
Complex

Sentences

\section{Coordinating Conjunctives Subordinating}

Conjunctions Adverbs Conjunctions

\begin{tabular}{|c|c|c|c|}
\hline Addition & and & $\begin{array}{l}\text { likewise, moreover } \\
\text { then, besides }\end{array}$ & \\
\hline $\begin{array}{l}\text { Contrast } \\
\text { or } \\
\text { Contradict } \\
\end{array}$ & $\begin{array}{l}\text { but } \\
\text { yet }\end{array}$ & $\begin{array}{l}\text { however, instead } \\
\text { nevertheless, still }\end{array}$ & \\
\hline $\begin{array}{l}\text { Cause (or } \\
\text { Reason } \\
\text { and Effect } \\
\text { (or } \\
\text { Result) }\end{array}$ & $\begin{array}{l}\text { so } \\
\text { for }\end{array}$ & $\begin{array}{l}\text { consequently, hence, } \\
\text { thus, accordingly } \\
\text { therefore }\end{array}$ & $\begin{array}{l}\text { because } \\
\text { as, since } \\
\text { so that } \\
\text { ir order that }\end{array}$ \\
\hline $\begin{array}{l}\text { Alternati- } \\
\text { ves }\end{array}$ & or, nor & otherwise & \\
\hline Condition & & & $\begin{array}{l}\text { if, as if } \\
\text { even if } \\
\text { unless, } \\
\text { though } \\
\text { as though } \\
\text { even though }\end{array}$ \\
\hline Time & & $\begin{array}{l}\text { meanwhile } \\
\text { subsequently } \\
\text { then, now } \\
\text { thereafter } \\
\text { eventually } \\
\text { presently }\end{array}$ & \\
\hline Place & & & $\begin{array}{l}\text { where } \\
\text { wherever }\end{array}$ \\
\hline
\end{tabular}


Rule: A comma must be placed before the coordinating conjunction when it is used to join sentences. Example: My mother sold her house, yet she can't help regretting it. Rule: When a conjunctive adverb is placed immediately between two sentences, a semicolon precedes and a comma often follows the connective. Example: The air conditioning system broke down; nevertheless, the student enjoyed his sentence combining exercises.

Rule: When the idea of lesser importance is placed first, a comma separates it from the second sentence; otherwise, no commas are needed. Example: Because Steve attended his English classes, he passed the exam.

Estos tipos de esquemas son muy útiles. El alumno no tiene por qué memorizar las conectivas.

\section{Activity 1: Coordinating Conjunctions}

1. The temperature was five degrees below normal in January. (so)

Home heating bills rose by ten percent.

The temperature was five degrees below normal in January, so home heating bills rose by ten percent.

\section{Activity 2: Conjunctive Adverbs}

1. Patricia returned the wallet to the store owner. (subsequently)

She received a reward of ten dollars.

Patricia returned the wallet to the store owner; subsequently, she received a reward of ten dollars.

\section{Activity 3. Subordinating Conjunctions}

1. I am not cleaning your bedroom. (while)

You read the newspaper.

I am not cleaning your bedroom while you read the newspaper.

En este tipo de ejercicio, el alumno tendrá que leer las oraciones y decidir qué conectiva usar.

\section{Activity 4: Connectives Establishing the Relationship of Addition}

1. Peter washed the dishes.

Susan put them away.

Peter washed the dishes; then, Susan put them away. 
Activity 5: Connectives Establishing the Relationship of Contrast or Contradiction

1. Tonny has a lot of homework.

He went to the football game.

\section{Activity 6: Connectives Establishing the Relationship of Cause and Effect}

1. Teresa knew the answers to the English test.

Her English boyfriend had helped her with her homework last night.

\section{Activity 7: Cued Connectives}

1. My sister is afraid of heights. (Condition)

She sometimes flies on airplanes.

Even though mi sister is afraid of heights, she sometimes flies on airplanes.

2. Mrs. Blackwell took a long vacation. (Contrast)

Her health did not improve.

Mr. Benson took a long vacation, but his health did not improve.

\section{Activity 8: Without cues}

1. Sarah went on a diet.

Even her boyfriend made fun of her weight. (Relationship:

\section{Activity 9: Combining ideas in a Narrative}

All cues have been removed from the story. In order to create an effective paragraph, combine those sentences that you feel can be joined by conjunctives.

The blizard during the winter of 1978 crippled New England.

People found themselves stuck in their houses for days.

They become grouchy and restless.

They had "cabin fever."

Newspaper experts claimed cures for this desease.

TV commentators documented the illness.

After the crippling blizard that hit New England during the winter of 1978, people found themselves confined to their houses for days. When the people became grouchy and restless, they had "cabin fever." Newspaper experts claimed cures for this desease, whereas the TV commentators documented the illness. 


\section{IDEAS ATTACHED TO NOUNS}

1. relative clauses

2. appositives

3. participial phrases

\section{Activity 1: Cued Activity set (relative clauses)}

1. The black cat was still on the porch this morning.

The black cat had appeared at our door last night. (,which ...,)

The black cat, which had appeared at our door last nigh, was still on the porch this morning.

Activity 2: Who, Whose, Whom (relative clauses)

1. After he won the election, Barry gave a thank-you speech to all the workers.

The enthusiasm of the workers had contributed so much to the campaign.

\section{Activity 3: Which, That, Commas (relative clauses)}

1. The term "Sentence Combining" applies to a technique.

Teachers use the technique to improve student's writing.

\section{Activity 4: Noun-Cued Sentences (appositives)}

1. Lisa painted each fingernail a different color

Lisa is the class clown.

Lisa, the class clown, painted each fingernail a different color.

\section{Activity 5: Cued Activity (participial phrases)}

1. Mary waits for the English class to end. (-ing ...)

Mary anxiously watches the clock.

Waiting for the English class to end, Mary anxiously watches the clock.

2. The sentence combining exercise challenged the student. (-ed ...)

The student forgot to watch her favorite TV show.

Se pueden realizar también muchos ejercicios con frases de infinitivo, de gerundio, sustantivas, paralelas, comparativas, etc. Veamos unos últimos ejercicios: 


\section{Activity 1: Combining to Form Infinitives}

1. Carlos wanted SOMETHING.

He would improve his grade in English.

Carlos wanted to improve his grade in English.

\section{Activity 2: Gerund Phrases}

1. I jog five miles a day.

I have improved my health by this activity.

I have improved my health by jogging five miles a day.

2. Ann read Neruda's poem aloud.

The recital moved most of the audience to tears.

Ann's reading aloud of Neruda's poems moved the audience to tears.

\section{Activity 3: That Noun Clauses}

1. The American writer Robert Frost wrote about New England landscape.

SOMETHING has proven to be very pleasant for many readers.

That the American writer Robert Frost wrote about New England landscape has proven to be very pleasant for many readers.

Por último hay que considerar que la práctica de combinación de oraciones no deben ser un fin en sí misma, sino más bien una forma de conseguir un buen dominio de la sintaxis de la oración, algo crucial para comprender los principios de organización de gobiernan al párrafo y al ensayo, antes de pasar a la composición libre. 